\title{
Challenges in Designing Inter-usable Systems
}

\author{
Ville Antila ${ }^{1}$ and Alfred Lui ${ }^{2}$ \\ ${ }^{1}$ VTT Technical Research Centre of Finland, Kaitoväylä 1, Oulu, Finland \\ ville.antila@vtt.fi \\ ${ }^{2}$ Fjord, 19 Margaret Street, London, United Kingdom \\ alfred.lui@fjord.co.uk
}

\begin{abstract}
Interactive systems are increasingly interconnected across different devices and platforms. The challenge for interaction designers is to meet the requirements of consistency and continuity across these platforms to ensure the inter-usability of the system. In this paper we investigate the current challenges the designers are facing in the emerging fields of interactive systems. Through semi-structured interviews of 17 professionals working on interaction design in different domains we probed into the current methodologies and the practical challenges in their daily tasks. The identified challenges include but are not limited to: the inefficiency of using low-fi prototypes in a lab environment to test inter-usability and the challenges of "seeing the big picture" when designing a part of an interconnected system.
\end{abstract}

Keywords: Interaction design, cross-platform systems, inter-usability.

\section{Introduction}

While the amount and diversification of computing devices is increasing the boundaries between the systems that can be accessed with them is blurring. Users expect to have access to the same applications and services with a multitude of devices and expect that this process is highly optimized to support the capabilities of the device at hand. In addition to this optimization, applications are expected to support efficient flow of interaction and coherence across the different user interfaces [10]. Today's products are becoming increasingly ubiquitous systems, hybrids of hardware, software and services [2]. It is not yet very clear what design and evaluation techniques should be used to design these products.

Nevertheless, these are the challenges that designers of applications and services are facing today. In this paper we take a look at the challenges in designing interusable systems from the designers and developers perspective. With inter-usability we mean the usability and user experience across the different user interfaces of a given system (also including inter-device interactions, similarly to [1]). The added complexity in the system requirements translate to added complexity in interaction design as well as challenges in evaluating the overall usability of the system.

To identify some of the specific challenges that practitioners of interaction design are facing, we conducted semi-structured interviews with 17 specialists in interaction and user interface design, working with services and applications designed 
for different levels of inter-usability. The application domains span from experimental products and prototypes mixing physical and digital elements to mass-market consumer products with user interfaces for both dedicated mobile applications and Webbased services. We asked the designers of these systems about the design and development processes they use, what kind of methodologies they use and what are the main challenges they face in their daily work. Based on the interviews we analyzed the specific challenges and situated them to the different stages of the design process to be able to refine the requirements of emerging design methodologies and tools.

\section{Background}

We are starting to see an increasing amount of interconnected devices and services that will require new design methodologies and new kind of understanding to make them (inter) usable [3]. The understanding of challenges in designing interconnected and inter-usable systems as opposed to traditional interaction design has recently caught more attention because of the proliferation of cloud-based, multiple touchpoint service. Denis and Karsenty [1] propose a conceptual framework for interusability. The design principles address inter-device consistency, transparency and adaptability as well as knowledge and task continuity. They describe continuity in cross-platform systems as shared memory between the system and the user. In an inter-usable system the user can recover the state of operation and continue the task after a transition from one device to another. Similarly, Wäljas et al. [10] introduce a conceptual framework for cross-platform user experience. They argue that the central elements for cross-platform user experience are:

- Composition: How different platforms (applications and devices) within a system relate to each other. This can be about role allocation of different devices, distribution of functionality or modularity of functionality

- Continuity: Interoperability is supported by carrying out transitions between platforms, e.g. seamless synchronization of data and content, explicitly supporting users in migrating tasks across platforms by proposing linkages to other devices in the system

- Consistency: Keeping the user experience coherent across multiple platforms to assist the Continuity element of the cross-platform UX. This can be perceptual (look and feel), semantic (symbols and terminology) and syntactic (interaction logic).

Inter-usable systems are composed of applications designed for different platforms. The different applications can support different activities within the system and can interact with different service "touch-points". The relation between these applications can have different requirements depending on whether the actual service is just multichanneled through these applications or whether the different functionalities are actually distributed amongst them (whether the different platforms have a distinct purpose in the system) [8].

The current methodologies for designing and evaluating interactive systems are usually limited to evaluating systems on a single platform or device, and do not usu- 
ally take into consideration the inter-usability aspects of cross-platform consistency and continuity. To address the challenge of designing user interface for multiple platforms there has been ongoing research on conceptual frameworks (metaphors and interaction styles) [7], design frameworks (patterns, widgets, UI component toolkits) $[5,6]$, application frameworks (both design-time and run-time support) [4] and validation frameworks (usability techniques for testing multiple user interfaces) [7]. However, often there's a gap between frameworks, available methods and tools, and the practical needs of practitioners [9]. In this paper we take a look at the specific challenges and requirements for the methods and tools from a practitioner's perspective and situate the current state of the art based on these requirements.

\section{Research Approach}

To identify current challenges of interaction design, we carried out semi-structured interviews with 17 professionals from 10 different organizations. The organizations spanned from major consumer product manufacturers to small design firms and startups. The interviewees included 6 interaction designers, 4 researchers in the field of user-centered design, 2 freelancer designers, and 3 founders of start-ups in the field of ubiquitous computing as well as one application engineer and one technical director. The interviews were conducted face to face and over the phone and each interview lasted about one hour. The interviewees were recruited through personal connections and were selected based on the knowledge of their work. The criterion for selection was that the work includes components, which are interconnected with some level of measured usability.

During the conversations we first asked the interviewees about their background, the type of company they were working for, what kind of products they were designing and the typical users of those products. We also asked the interviewees about the common design models and methodologies they use in their work. We subsequently probed into the possible challenges and difficulties they face in applying these methodologies in their work (regarding the suitability of methodologies for the given tasks). In addition to the structured questions we developed follow-up conversations to interesting answers, especially regarding the challenges the interviewees were facing.

All interviews were recorded and transcripts were made from the recordings. The transcripts were coded using a set of themes: design process, usage of UI methodologies and biggest challenges. The challenges were also coded into clusters based on the design process phase. The individual challenges were also clustered together to find common themes of challenges. We describe the main findings and analysis in the following section.

\section{Findings}

The most used UI design methodologies identified by the interviews were iterative design and rapid prototyping. Nevertheless when probing to the actual practices they seem to depend heavily on the team and company size. In small design firms, which mostly work on custom prototypes and products, the emphasis is on rapidly 
implementing ideas, which then translates to minimum use of traditional usercentered research practices and formal usability testing. In bigger companies targeting products for mass-markets, the use of rigorous processes usually translates to welldefined use of UI design methodologies. In the latter cases iterative and rapid prototyping is preferred, since it allows them to have early user involvement and avoid UI design changes in later phases when major changes to the product design is usually too late. We found this same division also applicable to the identified challenges (the identified challenges are listed in Table 1).

Based on the interviews we found a set of challenges that the designers were facing in their work. Some of these challenges were quite universal, such as the challenges in time and communication. Therefore, those are not discussed further. In this paper we concentrate on the following identified challenges related to inter-usability:

- Development environment constraints: The limitations of current development environments (e.g. challenges in prototyping systems which include both hardware and software)

- Domain restrictions: The restrictions of applications domains and their implications to practical choices between technologies (e.g. deployment strategies in restricted domains like healthcare)

- Acquiring domain knowledge and user feedback: Challenges in acquiring the right domain knowledge and user feedback early enough (e.g. challenges in simulating systems which span different devices with different capabilities)

- Developing for multiple platforms: The challenges for multiple platforms span from specific challenges of developing different interactions to support different capabilities of devices to maintain the consistency and continuity of interaction in an interconnected system

Development environment constraints - In some interviews it was mentioned that the design phase and development phase should be easier to integrate. In small companies this can be done with close iteration and even using the same tools to design and prototype. In larger teams where design and implementation activities are more separated, UI component libraries can help establish this integration. This is often done implicitly, without any real integration on the tool-level. The designers often cannot test the components and UI flows interactively before the real implementation phase, which imposes difficulties to spot design faults enough early in the development.

"There are component libraries and design guidelines between product families, but these do not go all the way into the 'tools' -level. It would be useful to share the same common tools with developers to avoid the usual challenge [the designers face] 'this cannot be implemented on this platform'" - Interaction designer, UI designs for mobile and Web-based applications.

While these challenges are also observed in traditional design and development workflows, the lack of standard library and data protocols across platforms compound their impact on the efficiency between the teams. 
Domain restrictions - Some domains impose restrictions on the used technologies by using old versions of software platforms or by limiting the capabilities of installing new software. Also in some fields expert insights and inputs are crucial for a successful design but the inputs are hard to get because of tight schedules or policies. The limitations on the deployment of new technologies affect both the continuity and consistency of cross-platform solutions in that domain. This creates implications to the used technologies. Following example describes some of the challenges imposed by the healthcare domain:

"Many of users work in an environment where installation of new software or packages on their computers is restricted, for example by hospital IT departments. We have to work within these restrictions. For that reason we choose web based UI's for most of our projects because it does not require installation of software on the [devices] of the users" - Application engineer, user interfaces for applications in the domain of healthcare.

Acquiring domain knowledge and user feedback - Knowing the users and their needs is a major challenge identified in all interviews. Nevertheless the type and way of getting the knowledge from users may differ greatly by the type of product. In experimental projects by small companies the target group can be hard to define and involve in the early stage so the design process is very much driven by intuition and personal insights. In specific domains like health-care the challenge often is to find out what the real user needs are. This can be made difficult by the time restrictions of domain professionals.

When designing an interconnected system another challenge is the inefficiency of user testing in lab environment. In many cases the interviewees identified early user feedback as important or vital, but also noted that they cannot get feedback until they have a functional prototype which can be tested in the field. Testing usability of solutions to accommodate change in context is particularly hard due to the lack of standardized research framework and analysis methods.

"A lot of the things we are changing, we will do more of a [...] we put it out there and see if people are using it, we don't spend that time doing a full usability type of thing. Moving towards what the 'Google model' is like, they almost put up the lab version or they have a beta version and people just use it, get the feedback and just improve it." - Co-founder of a start-up on professional sports tracking technology.

Targeting multiple platforms - The challenge in designing for multiple platforms is the different capabilities and user interaction metaphors they may have. There is no easy way to meet this challenge. Part of the design always has to be adjusted to the target device on its own, but re-usability of user interfaces across the platforms is also needed for a unified look-and-feel when dealing with an interconnected system.

"As [the application] must run on several devices, and the devices have different capabilities in terms of display and user controls, it is difficult to design a UI that can be used as fast and easy on all the devices." - Technical director, mobile and ubiquitous gaming applications. 
Table 1. Identified challenges grouped by the design process phases

\begin{tabular}{|c|c|c|}
\hline Process phase & Identified activities & Identified challenges \\
\hline Early phase & $\begin{array}{l}\text { Iterative concept } \\
\text { creation and } \\
\text { evaluation, } \\
\text { Interviews, } \\
\text { Contextual inquiries, } \\
\text { Focus groups }\end{array}$ & $\begin{array}{l}\text { - Hard to evaluate concepts without functional } \\
\text { prototypes (simulation of interactive system) } \\
\text { Seeing the big picture when designing a part of a } \\
\text { interconnected service (often designers just } \\
\text { focus on part of the system which can create in- } \\
\text { consistencies and discontinuities between the } \\
\text { parts) }\end{array}$ \\
\hline Design phase & $\begin{array}{l}\text { Rapid prototyping } \\
\text { (e.g. paper), } \\
\text { Integration of design } \\
\text { and development } \\
\text { tools, } \\
\text { Task analysis }\end{array}$ & $\begin{array}{l}\text { Each failed experiment with physical objects } \\
\text { incurs material, labor and transportation costs } \\
\text { (unlike with fully digital products/services) }\end{array}$ \\
\hline $\begin{array}{l}\text { Development } \\
\text { phase }\end{array}$ & $\begin{array}{l}\text { Functional } \\
\text { prototyping, } \\
\text { Use of (in-house) } \\
\text { component libraries }\end{array}$ & $\begin{array}{l}\text { - None of the tools available today is sufficient to } \\
\text { build and test inter-usable systems } \\
\text { Basic tools such as IDEs, Flash and PCB design } \\
\text { tools are generic enough to fill the gap but by no } \\
\text { means efficient for designers who want to weave } \\
\text { digital data into physical materials }\end{array}$ \\
\hline $\begin{array}{l}\text { Evaluation } \\
\text { phase }\end{array}$ & $\begin{array}{l}\text { Expert reviews, } \\
\text { Usability testing, } \\
\text { Field trials }\end{array}$ & $\begin{array}{l}\text { - User testing of embedded devices and } \\
\text { interconnected services using low-fi prototypes } \\
\text { in a lab environment is inefficient } \\
\text { Difficult to evaluate the whole (interconnected) } \\
\text { system; evaluation of separated parts does not } \\
\text { necessarily correspond to good overall (inter) } \\
\text { usability }\end{array}$ \\
\hline
\end{tabular}

\section{Analysis and Implications for Design Methodologies}

Based on the information gathered from the interviews we analyzed the possible needs for refinement of methods and tools. We identified three basic types of needs from the interviews. There is currently a need for better integration between the design and development tools. A number of interviewees identified that it is challenging to test designs on different platforms enough early in the design process. They identified a need for "mash-up" type development environment where the composition and continuity of an inter-usable system could be better tested. Another challenge, which could benefit from better tools, is the possibility to link smaller design tasks into the "big picture". This can be especially difficult in systems incorporating several different user interfaces for different devices and platforms. This cross-platform design support also needs different methodologies than more traditional single platform systems. The current design methodologies do not take into the consideration the need to support effective transitions and interaction metaphors across platforms. The implications of findings are described more in the Table 2. 
Table 2. Implications of findings to requirements for design methods and tools

\begin{tabular}{|c|c|c|}
\hline Identified need & Description & Requirements for methods and tools \\
\hline $\begin{array}{l}\text { Support for "seeing } \\
\text { the big picture"- } \\
\text { how the design fits in } \\
\text { the whole system }\end{array}$ & $\begin{array}{l}\text { Consistency and continuity is } \\
\text { important in cross-platform } \\
\text { user experience, the current } \\
\text { design methods and tools do } \\
\text { not offer support for evaluat- } \\
\text { ing this }\end{array}$ & $\begin{array}{l}\text { Early prototyping through } \\
\text { simulation } \\
\text { Evaluation metrics to test } \\
\text { consistency (semantic and } \\
\text { syntactic) and continuity in } \\
\text { cross-platform and cross-device } \\
\text { interactions }\end{array}$ \\
\hline $\begin{array}{l}\text { Integration between } \\
\text { design and develop- } \\
\text { ment tools }\end{array}$ & $\begin{array}{l}\text { There are challenges in inte- } \\
\text { gration of design and imple- } \\
\text { mentation. The currently } \\
\text { available tools are not suffi- } \\
\text { cient to build and test inter- } \\
\text { usable systems (such as } \\
\text { services with interfaces for } \\
\text { both cloud and dedicated } \\
\text { devices) }\end{array}$ & $\begin{array}{l}\text { Ability to test or "mash-up" the } \\
\text { composition of interconnected } \\
\text { systems (e.g. distribution and } \\
\text { composition of functionalities } \\
\text { between the cloud and dedicated } \\
\text { devices) } \\
\text { - Support for rapid prototyping }\end{array}$ \\
\hline $\begin{array}{l}\text { Refinement of } \\
\text { evaluation methods } \\
\text { and metrics to test } \\
\text { inter-usability }\end{array}$ & $\begin{array}{l}\text { There are challenges in } \\
\text { evaluating interconnected } \\
\text { systems early enough, and to } \\
\text { measure inter-usability. } \\
\text { There is also a need for early } \\
\text { evaluation of interaction } \\
\text { metaphors which translate } \\
\text { between the different do- } \\
\text { mains and user interfaces } \\
\text { designed for different plat- } \\
\text { forms }\end{array}$ & $\begin{array}{l}\text { Evaluation methods and metrics to } \\
\text { support inter-usability, taking into } \\
\text { account both the composition of } \\
\text { functionalities and the continuity } \\
\text { of interaction } \\
\text { Design guidelines to support } \\
\text { semantic consistency across plat- } \\
\text { forms (the use of metaphors etc.) } \\
\text { Ability to use efficiency measures } \\
\text { to validate inter-usability of cross- } \\
\text { platform interactions }\end{array}$ \\
\hline
\end{tabular}

The implications of our findings can be used as an indicator of growing needs for measuring and testing the inter-usability of interconnected systems. It is clear that the tools and methods in design and development at their current states do not sufficiently address these needs for cross-platform design. Based on our findings the specific needs lie in the design and evaluation phases where we found needs for both rapid prototyping tools for interconnected systems as well as better evaluation metrics to test the composition, consistency and continuity aspects of cross-platform user experience [10]. Because of that, the designing and developing for such systems currently involve a great deal of intuition and trial-and-error.

Another factor that contributes to the challenge is that devices themselves no longer offer user standardized means of manipulating information. Mobile phones, the epitome of multi-platform, cross-context devices, have morphed from a combination of a 12-digit pad, a set of 5-way navigation key and a small display to a touchsensitive screen that can offer any number of controls. Going further, interconnected objects that are believed to embed information access into our environments in the near future can come in theoretically limitless number of forms. 


\section{Conclusions}

In this paper we have presented the analysis and implications of findings identified through interviews of professionals in interaction design. We identified a set of specific challenges that the practitioners face on different stages of the design process. We also identified needs for further refinements of methodologies to overcome the challenges imposed by requirements of inter-usability. We acknowledge these results as preliminary and foresee a need for further research on translating the identified challenges and needs to refinements on methods and tools to support these challenges in practice. Nevertheless we see this work as an important step towards understanding the needs of inter-usability better from the point-of-view of professionals dealing with the practical challenges of designing interconnected systems and services.

Acknowledgements. This work has been supported by the SMARCOS Artemis project. We would like to thank Privender Saini, Marloes van der Hout, Josu Cobelo, Jorge Rodriguez and Guido Galeazzi for their contributions to the interviews and analysis. We also acknowledge the comments and suggestions from Tine Lavrysen regarding this paper.

\section{References}

1. Denis, C., Karsenty, L.: Inter-Usability of Multi-Device Systems - A Conceptual Framework. In: Seffah, A., Javahery, H. (eds.) Multiple User Interfaces: Cross-Platform Applications and Context-Aware Interfaces. John Wiley \& Sons, Ltd., Chichester (2004)

2. Kuniavsky, M.: Observing the user experience: a practitioner's guide to user research. Morgan Kaufmann, San Francisco (2003)

3. Le Voi, H., Light, A., Rowland, C.: Towards interusability; HCI for cloud computing and embedded devices. In: Proceedings of Designing Interaction for the Cloud Workshop in CHI 2011 (2011)

4. Melchior, J., Grolaux, D., Vanderdonckt, J., Van Roy, P.: A toolkit for peer-to-peer distributed user interfaces: concepts, implementation, and applications. In: Proceedings of the 1st ACM SIGCHI Symposium on Engineering Interactive Computing Systems, EICS 2009, p. 69. ACM, New York (2009)

5. Nilsson, E.: Combining compound conceptual user interface components with modelling patterns-a promising direction for model-based cross-platform user interface development. In: Forbrig, P., Limbourg, Q., Urban, B., Vanderdonckt, J. (eds.) DSV-IS 2002. LNCS, vol. 2545, pp. 104-117. Springer, Heidelberg (2002)

6. Seffah, A., Forbrig, P., Javahery, H.: Multi-devices "Multiple" user interfaces: development models and research opportunities. Journal of Systems and Software 73(2), 287-300 (2004)

7. Seffah, A., Javahery, H.: Multiple User Interfaces: Cross-Platform Applications and Context-Aware Interfaces. John Wiley \& Sons, Ltd., Chichester (2004)

8. Segerståhl, K., Oinas-Kukkonen, H.: Distributed user experience in persuasive technology environments. In: de Kort, Y.A.W., IJsselsteijn, W.A., Midden, C., Eggen, B., Fogg, B.J. (eds.) PERSUASIVE 2007. LNCS, vol. 4744, pp. 80-91. Springer, Heidelberg (2007)

9. Väänänen-Vainio-Mattila, K., Roto, V., Hassenzahl, M.: Towards Practical User Experience Evaluation Methods. In: International Workshop on Meaningful Measures: Valid Useful User Experience Measurement (VUUM), p. 19 (2008)

10. Wäljas, M., Segerståhl, K., Väänänen-Vainio-Mattila, K., Oinas-Kukkonen, H.: Cross-platform service user experience: a field study and an initial framework. In: Proceedings of the 12th International Conference on Human Computer Interaction with Mobile Devices and Services, MobileHCI 2010, p. 219. ACM, New York (2010) 